\title{
Enhanced tunable narrow-band THz emission from laser-modulated electron beams
}

\author{
D. Xiang and G. Stupakov \\ SLAC National Accelerator Laboratory, Menlo Park, California 94025, USA
}

(Received 4 June 2009; published 10 August 2009)

\begin{abstract}
We propose and analyze a scheme to generate enhanced narrow-band terahertz (THz) radiation through down-conversion of the frequency of optical lasers using laser-modulated electron beams. In the scheme the electron beam is first energy modulated by two lasers with wave numbers $k_{1}$ and $k_{2}$, respectively. After passing through a dispersion section, the energy modulation is converted to density modulation. Because of the nonlinear conversion process, the beam will have density modulation at wave number $k=n k_{1}+$ $m k_{2}$, where $n$ and $m$ are positive or negative integers. By properly choosing the parameters for the lasers and dispersion section, one can generate density modulation at $\mathrm{THz}$ frequency in the beam using optical lasers. This density-modulated beam can be used to generate powerful narrow-band THz radiation. Since the $\mathrm{THz}$ radiation is in tight synchronization with the lasers, it should provide a high temporal resolution for the optical-pump THz-probe experiments. The central frequency of the THz radiation can be easily tuned by varying the wavelength of the two lasers and the energy chirp of the electron beam. The proposed scheme is in principle able to generate intense narrow-band $\mathrm{THz}$ radiation covering the whole $\mathrm{THz}$ range and offers a promising way towards the tunable intense narrow-band $\mathrm{THz}$ sources.
\end{abstract}

DOI: 10.1103/PhysRevSTAB.12.080701

PACS numbers: 07.57.Hm, 41.60.Cr, 41.75.Ht

\section{INTRODUCTION}

There is growing interest in generating powerful terahertz $(\mathrm{THz})$ radiation to fill the frequency gap ranging from 0.1 to $30 \mathrm{THz}$ which has wide applications in nondestructive imaging and spectroscopic studies of materials and molecules (for a recent review, see Refs. [1-3]). Considerable efforts have been made in both the laser community $[4,5]$ and the accelerator community [6-11] to fill the gap. Using laser technologies to generate $\mathrm{THz}$ radiation has the advantage of being compact, but typically with a limited peak power. The accelerator-based $\mathrm{THz}$ sources are capable of providing intense $\mathrm{THz}$ radiation with $\mathrm{MW}$ peak power and are particularly suited for nonlinear optical phenomena studies and nonlinear spectroscopy measurements [8].

Accelerator-based $\mathrm{THz}$ sources typically fall into two categories. The first category uses ultrashort electron beam to generate intense broad-band $\mathrm{THz}$ radiation which is very suitable for single-cycle nonlinear optical phenomena studies [8]. One simple method to generate broad-band $\mathrm{THz}$ radiation is to compress the beam to picosecond (ps) or sub-ps level and the $\mathrm{THz}$ radiation may be generated from transition radiation, diffraction radiation, synchrotron radiation, undulator radiation, etc. However, it appears difficult to extend the radiation to $>5 \mathrm{THz}$ which would require compressing the beam to sub-100 fs level. For the moderately relativistic beam $(\sim 50 \mathrm{MeV})$, the shortest bunch length is typically limited by the second order effects in the bunch compression and the space charge effects. Also a large compression ratio typically involves a serious degradation of the beam quality. There also exists many applications (e.g. THz imaging) that require (or could benefit from) a powerful narrow-band $\mathrm{THz}$ radiation. The second category typically aims to provide such kinds of narrow-band $\mathrm{THz}$ radiation using bunched electron beam with a density modulation repeated at $\mathrm{THz}$ frequency $[9,10]$. One method is to use a train of laser pulses to illuminate the photocathode to generate a train of electron beams repeated at $\mathrm{THz}$ frequency and the bunch trains can be used to generate narrow-band $\mathrm{THz}$ radiation when they are accelerated to relativistic energy [10]. However, the space charge force tends to wash out the longitudinal structure during the acceleration process and may limit the application to a relatively low current beam.

To mitigate the space charge effect, it is desirable to generate density modulation in electron beams when they are at relativistic energy. In Ref. [9] a laser is first shaped to have a quasisinusoidal envelope at $\mathrm{THz}$ frequency and then used to generate energy modulation in the relativistic electron beam. After passing through a dispersion section, the energy modulation is converted to density modulation. This method has been demonstrated to be very effective in generating tunable narrow-band $\mathrm{THz}$ radiation.

In this paper, we propose and analyze a scheme to generate intense tunable narrow-band $\mathrm{THz}$ radiation via laser-electron interactions. The proposed scheme is similar to that used in the echo-enabled harmonic generation freeelectron laser $[12,13]$ except that here we only have one dispersion section and the aim is to down-convert the frequency of the lasers to generate $\mathrm{THz}$ modulation in the relativistic beam. The scheme is also analogous to the 
difference-frequency generation (DFG) of two lasers [5], with the relativistic electron beam being the nonlinear medium.

In the scheme the electron beam is first energy modulated by two lasers with wave number $k_{1}$ and $k_{2}$, respectively. After passing through a dispersion section, the energy modulation is converted to density modulation. Because of the nonlinear conversion process, the beam will have density modulation at wave number $k=n k_{1}+$ $m k_{2}$, where $n$ and $m$ are positive or negative integers. By properly choosing the parameters for the lasers and dispersion section, one may generate density modulation at $\mathrm{THz}$ frequency in the beam using optical lasers. This densitymodulated beam can be used to generate powerful narrowband $\mathrm{THz}$ radiation. The central frequency of the $\mathrm{THz}$ radiation can be easily tuned by varying the wavelengths of the lasers and the energy chirp of the electron beam. The proposed scheme is in principle able to generate $\mathrm{THz}$ radiation covering the whole $\mathrm{THz}$ range. In addition to generation of $\mathrm{THz}$ radiation, the density-modulated beam can also be used to enhance the performances of infrared free-electron lasers [14] and plasma wakefield accelerators $[15,16]$.

\section{GENERATION OF DENSITY MODULATION IN ELECTRON BEAM: THEORY}

The scheme for generating density modulation in the electron beam is shown in Fig. 1. It consists of two modulators and one dispersion section. Two lasers with different wavelengths are used to modulate the beam energy in the undulators (modulator 1 and modulator 2). It is worth pointing out that, when the wavelengths of the two lasers are close and the number of undulator periods $N_{p}$ is small, i.e. $2\left|k_{1}-k_{2}\right| /\left(k_{1}+k_{2}\right) \ll 1 / N_{p}$, one can eliminate the second modulator and modulate the beam energy with both frequencies by sending the two laser beams simultaneously with the electron beam into the first undulator. In what follows, however, we will assume the more general setup shown in Fig. 1 which, in principle, allows one to have arbitrary values $k_{1}$ and $k_{2}$.

We assume an initial Gaussian beam energy distribution with an average energy $E_{0}$ and the rms energy spread $\sigma_{E}$, and use the variable $p=\left(E-E_{0}\right) / \sigma_{E}$ for the dimension-

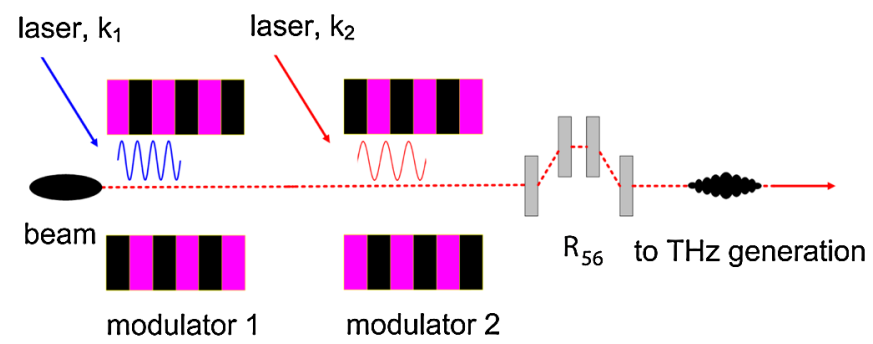

FIG. 1. (Color) Scheme for generation of density modulation in electron beam. less energy deviation of a particle. Since the bunch length is typically much larger than the wavelength of the modulation, we neglect the variation of the beam current within the bunch and assume a longitudinally uniform beam. The initial longitudinal phase space distribution can then be written as $f_{0}(p)=N_{0}(2 \pi)^{-1 / 2} e^{-p^{2} / 2}$, where $N_{0}$ is the number of electrons per unit length of the beam. After passage through the two undulators, the beam energy is modulated with the amplitude $\Delta E_{1}$ and $\Delta E_{2}$, so that the final dimensionless energy deviation $p^{\prime}$ is related to the initial one $p$ by the equation $p^{\prime}=p+A_{1} \sin \left(k_{1} z\right)+$ $A_{2} \sin \left(k_{2} z+\phi\right)$, where $A_{1}=\Delta E_{1} / \sigma_{E}, A_{2}=\Delta E_{2} / \sigma_{E}$, $k_{1}$ and $k_{2}$ are the wave numbers of the laser in the first and second modulator, respectively, $z$ is the longitudinal coordinate in the beam, and $\phi$ is the phase difference of the second laser. The distribution function after the interaction with the laser becomes

$$
\begin{aligned}
f_{1}(z, p)= & \frac{N_{0}}{\sqrt{2 \pi}} \exp \left[-\frac{1}{2}\left[p-A_{1} \sin k_{1} z\right.\right. \\
& \left.\left.-A_{2} \sin \left(k_{2} z+\phi\right)\right]^{2}\right] .
\end{aligned}
$$

Sending then the beam through the dispersion section with the dispersive strength $R_{56}$ converts the longitudinal coordinate $z$ into $z^{\prime}, z^{\prime}=z+R_{56} p \sigma_{E} / E_{0}$ (where $p$ now refers to the value at the entrance to the first dispersion section), and makes the distribution function

$$
\begin{aligned}
f_{f}(z, p)= & \frac{N_{0}}{\sqrt{2 \pi}} \exp \left[-\frac{1}{2}\left[p-A_{1} \sin \left(k_{1} z-B p\right)\right.\right. \\
& \left.\left.-A_{2} \sin \left(k_{2} z-K B p+\phi\right)\right]^{2}\right]
\end{aligned}
$$

where $B=R_{56} k_{1} \sigma_{E} / E_{0}$ and $K=k_{2} / k_{1}$.

After passing through the dispersion section, the beam will have density modulation at various frequencies. Integration of (2) over $p$ gives the beam density $N(z)$ which contains various Fourier wave numbers $k_{n, m}=(n+$ $m K) k_{1}$ in it (see [12,13]). The bunching factors $b_{n, m}$ for

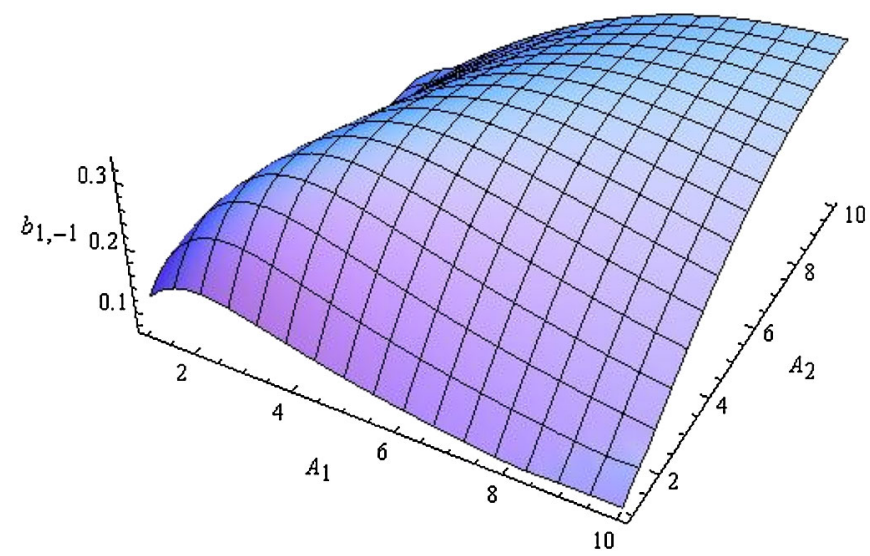

FIG. 2. (Color) The maximized bunching factor $b_{1,-1}$ as a function of the modulation amplitudes $A_{1}$ and $A_{2}$. 


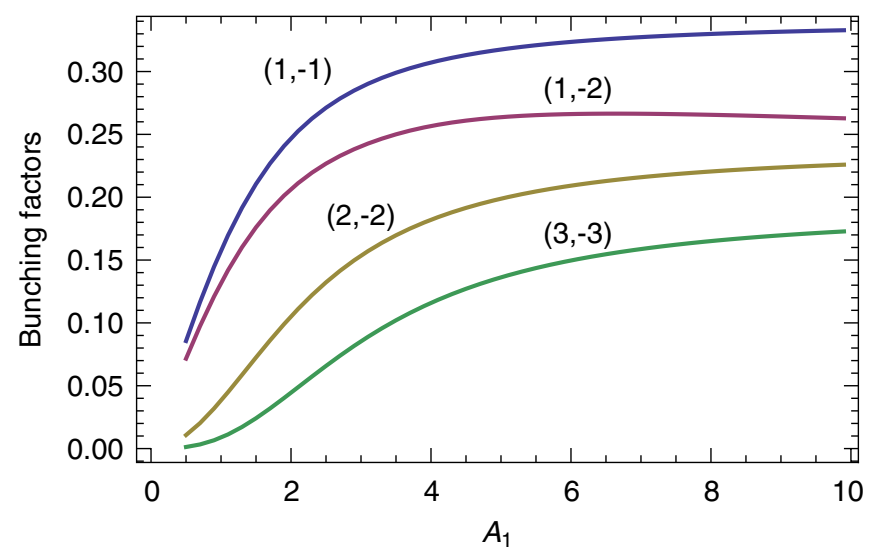

FIG. 3. (Color) The maximized bunching factors $b_{1,-1}, b_{1,-2}$, $b_{2,-2}$, and $b_{3,-3}$ as functions of the modulation amplitudes $A_{1}$. The pairs $(n, m)$ for each curve are shown in the picture.

each harmonic are defined as $\left\langle\left[N(z) / N_{0}\right] e^{-i k_{n, m} z}\right\rangle$, where the brackets denote averaging over the longitudinal coordinate $z$. After some mathematical manipulations the bunching factor is found to be independent of the laser phase difference and can be written as $[12,13]$

$$
\begin{aligned}
b_{n, m}= & \mid J_{n}\left[(n+K m) A_{1} B\right] \\
& \times J_{m}\left[(n+K m) A_{2} B\right] e^{-(1 / 2)[(n+K m) B]^{2}} \mid .
\end{aligned}
$$

For the case where only one modulator is present, Eq. (3) reduces to the well-known formula for the bunching factor in a high-gain harmonic generation free-electron laser [17].

For given values of the amplitudes of the modulation $A_{1}$ and $A_{2}$ and the value $n+K m$, Eq. (3) can be maximized over the variable $B$ (the strength of the chicane). The result of such optimization for $n=1$ and $m=-1$ is shown in Fig. 2. One can see from that figure that the value of the bunching factor can be close to 0.3 when $A_{1}$ and $A_{2}$ are larger than 3 (and approximately equal to each other).

In another optimization procedure, we maximized the bunching factor (3) over the variables $A_{2}$ and $B$ (that is over the second amplitude of the modulation and the chicane strength) for a given value of $A_{1}$. The result of such optimization is shown in Fig. 3. From this figure, one can see that it is most effective to use two lasers with close wavelength to modulate the beam and generate $\mathrm{THz}$ frequency modulation at the difference frequency $(n=1$, $m=-1$ ), similar to the case in DFG of two lasers. For the case when $|n|+|m|>2$, generation of $\mathrm{THz}$ radiation from DFG becomes ineffective, because it involves the $(|n|+|m|)$ th order polarization of the nonlinear medium. However, for our scheme the bunching factor for $|n|+$ $|m|>2$ is still on the same order as that for $n=1$ and $m=$ -1 , so $\mathrm{THz}$ radiation with considerable intensity can still be generated.

To illustrate the dynamics occurring in the phase space of the beam, we show in Fig. 4 the phase space evolution of the beam for the optimized case with $K=1.1, A_{1}=3$, $A_{2}=3.46$, and $B=5.32$. The parameters are optimized
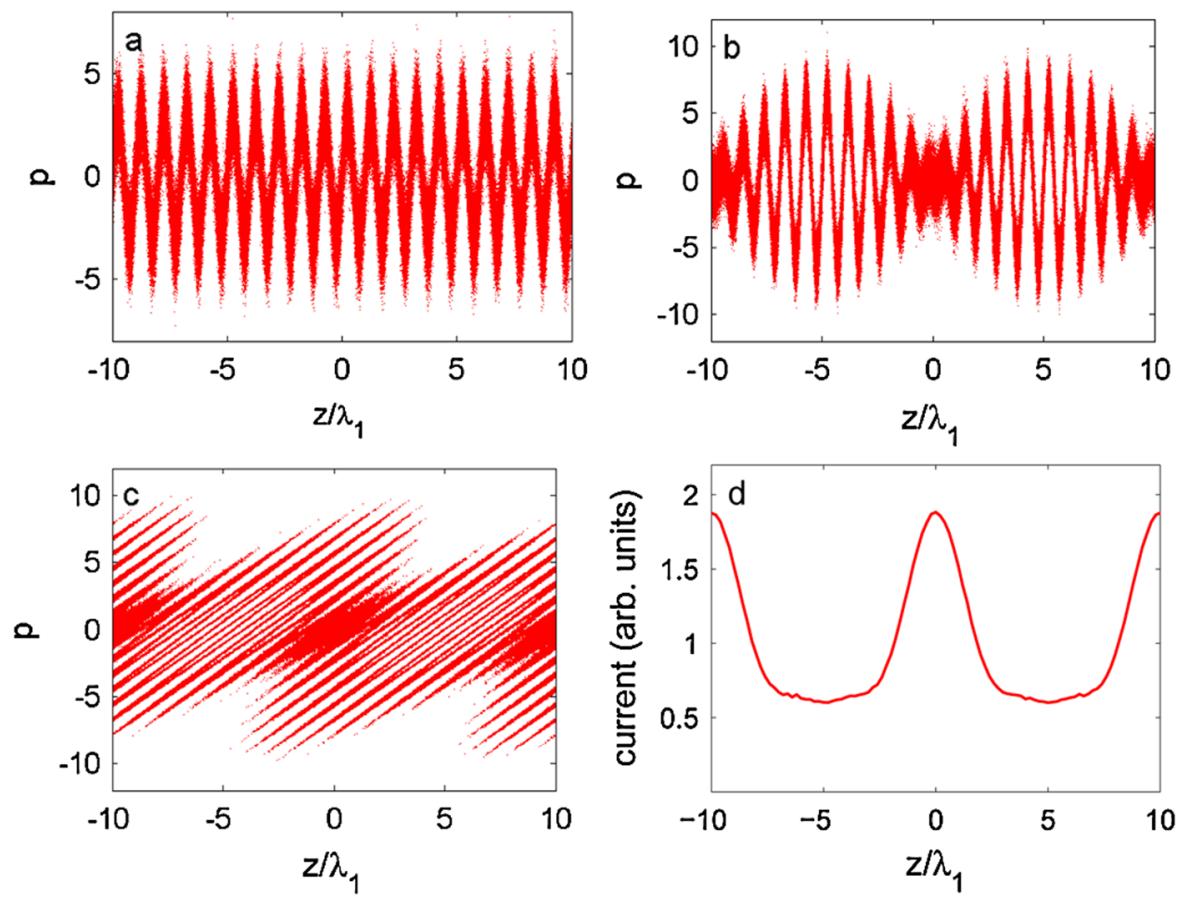

FIG. 4. (Color) The phase space of the beam optimized for the beam modulation with the wavelength equal to ten laser wavelengths $\lambda_{1}$ : (a) phase space at the exit of modulator 1; (b) phase space at the exit of modulator 2; (c) phase space at the exit of the dispersion section; (d) current distribution at the exit of the dispersion section. 
for the $n=1, m=-1$ harmonic with the wave number $k=0.1 k_{1}$. From Fig. 4(b) we see after modulator 2, the beam has an energy modulation envelope with the wave number $k=0.1 k_{1}$. The phase space is periodic with the period $10 \lambda_{1}$. After passing through the dispersion section, the complicated structure generated in the phase space produces beam modulation with the wavelength $\lambda=$ $10 \lambda_{1}$. The density modulation is clearly seen in Fig. 4(d) and the corresponding bunching factor is $b_{1,-1}=0.29$.

\section{GENERATION OF DENSITY MODULATION IN ELECTRON BEAM: SIMULATION}

Let us first consider an example where the wavelengths of the two lasers are close and their frequency difference lies in the $\mathrm{THz}$ region. We consider a Ti:sapphire laser which has a relatively broad bandwidth. The wavelengths of the lasers are assumed to be $\lambda_{1}=770 \mathrm{~nm}$ and $\lambda_{2}=$ $780 \mathrm{~nm}$, respectively. We assume a moderately relativistic electron beam with energy of $60 \mathrm{MeV}$. The beam has a Gaussian distribution with rms bunch length of $0.5 \mathrm{ps}$, peak current of $200 \mathrm{~A}$, and slice energy spread of $10 \mathrm{keV}$. Taking $n=1, m=-1$, and $A_{1}=5.00$, we found that the parameters that maximize the bunching factor for the differencefrequency radiation were $A_{2}=5.28$ and $B=27.20$ (corresponding to $R_{56}=20.00 \mathrm{~mm}$ ). The beam current and

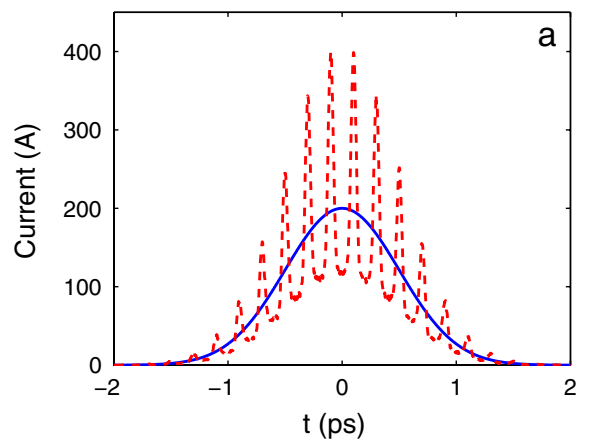

corresponding bunching factors for the case before and after laser modulation are shown in Fig. 5.

From Fig. 5 we can see that the frequency component of the unmodulated beam only extends to about $1 \mathrm{THz}$ while that for the laser-modulated beam has a strong spike at $5 \mathrm{THz}$ which equals the frequency difference of the two lasers. As can be seen in Fig. 3, the laser-modulated beam also contains considerable components at $10 \mathrm{THz}$ and $15 \mathrm{THz}$ which correspond to $n=2, m=-2$ and $n=3$, $m=-3$, respectively. As discussed in the previous section, the bunching factors for these high harmonics decrease as $|n|$ and $|m|$ increases.

A more interesting case is to use one laser with fixed wavelength and a second laser with tunable wavelength to generate $\mathrm{THz}$ radiation covering a wide frequency range. The second laser can be generated from optical parametric amplifiers (OPA). For example, the OPA pumped by Ti:sapphire laser at $\sim 800 \mathrm{~nm}$ can easily provide a tunable signal beam from 1.1 to $1.6 \mu \mathrm{m}$ [18]. Assuming the wavelength of the laser in the first modulator is $800 \mathrm{~nm}$ and that in the second modulator is $1560 \mathrm{~nm}$, taking $n=1$ and $m=$ -2 , the generated radiation has a frequency of $10 \mathrm{THz}$. With the same beam parameters as in Fig. 5 and assuming $A_{1}=3$, we found that the parameters that maximize the bunching factor were $A_{2}=5.74$ and $B=19.95$ (corresponding to $R_{56}=14.67 \mathrm{~mm}$ ). The beam current distribu-

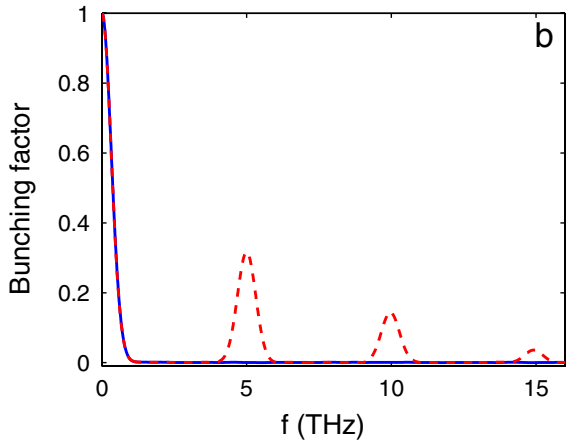

FIG. 5. (Color) (a) Initial current distribution (solid blue line) and that after the dispersion section (dashed red line). (b) Corresponding bunching factor for the initial current distribution (solid blue line) and that after the dispersion section (dashed red line).
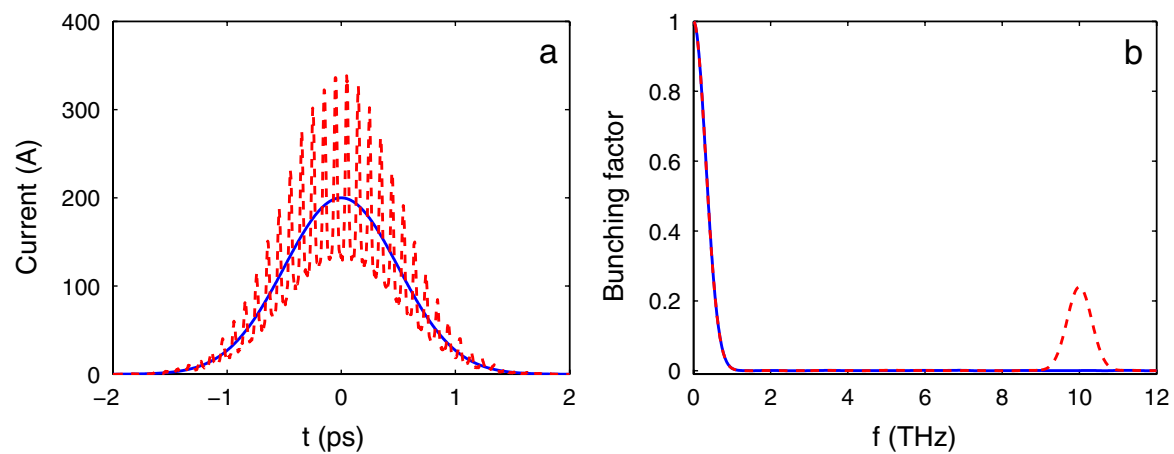

FIG. 6. (Color) (a) Initial current distribution (solid blue line) and that after the dispersion section (dashed red line). (b) Corresponding bunching factor for the initial current distribution (solid blue line) and that after the dispersion section (dashed red line). 
tion and bunching factor for the laser-modulated beam are shown in Fig. 6. As can be seen in Fig. 6, the lasermodulated beam was able to extend the $\mathrm{THz}$ radiation to $10 \mathrm{THz}$. Also there are considerable components at 20 and $30 \mathrm{THz}$ (not shown in Fig. 6).

It is worth pointing out that one can also generate $\mathrm{THz}$ this way with DFG (wave number of $2 k_{2}-k_{1}$ ), but it involves the third-order polarization [19] and the thirdorder nonlinear optical susceptibility is generally very small which limits the intensity of the generated $\mathrm{THz}$ radiation.

\section{GENERATION OF INTENSE NARROW-BAND THZ RADIATION}

There are many methods to generate $\mathrm{THz}$ radiation using relativistic electron beams, e.g., transition radiation (TR), diffraction radiation, synchrotron radiation, undulator radiation, etc. To illustrate a possible application of the proposed scheme in generating powerful narrow-band $\mathrm{THz}$, we consider TR which is typically generated when electron beam passes through a metallic foil. Assuming a round TR target with radius $r$, the TR spectral angular distribution for a single electron can be calculated as [20]

$$
\frac{d^{2} U}{d \Omega d \omega}=\frac{e^{2}}{4 \pi^{3} \epsilon_{0} c} \frac{\beta^{2} \sin ^{2} \theta}{\left(1-\beta^{2} \cos ^{2} \theta\right)^{2}}[1-\Upsilon(\mathrm{s})]^{2},
$$

where

$$
\begin{aligned}
\Upsilon(\mathrm{s})= & \frac{\mathrm{s}}{\beta \gamma \sin \theta}\left[J_{1}(\gamma \sin \theta \varsigma) K_{2}(\mathrm{~s})\right. \\
& \left.-\gamma \theta J_{2}(\gamma \sin \theta \mathrm{s}) K_{1}(\mathrm{~s})\right],
\end{aligned}
$$

where $\mathrm{s}=2 \pi r / \gamma \lambda, \theta$ is the polar angle, and $\gamma$ is the Lorentz factor.

Consider a relativistic electron beam with energy $60 \mathrm{MeV}$, rms bunch length of $1 \mathrm{ps}$, and bunch charge of $1 \mathrm{nC}$. The peak current is about $400 \mathrm{~A}$ and it can be achieved with a standard bunch compressor. The main parameters for the electron beam, the two lasers, and the undulators are listed in Table. I.

The two laser wavelengths are assumed to be 800 and $1560 \mathrm{~nm}$, respectively. Taking $n=1$ and $m=-2$, the generated radiation has a central frequency of $10 \mathrm{THz}$.

Assuming a round TR target with radius $5 \mathrm{~mm}$ and a collection angle of $0.2 \mathrm{rad}$, the calculated radiation energy density at various frequency is shown in Fig. 7. The suppression of the low frequency component in Fig. 7 is due to the finite size target effect of TR [20] which occurs when $\gamma \lambda_{r}>r$, where $\lambda_{r}$ is the radiation wavelength. The peak radiation intensity is about $3 \mu \mathrm{J} / \mathrm{THz}$ and peak power at $10 \mathrm{THz}$ is about $0.33 \mathrm{MW}$. This powerful narrow-band THz radiation should have wide applications in $\mathrm{THz}$ imaging, nonlinear spectroscopy, etc. Since the THz radiation is in
TABLE I. Main parameters for the laser-modulated $\mathrm{THz}$ source.

\begin{tabular}{lc}
\hline \hline Electron beam energy & $60 \mathrm{MeV}$ \\
Electron beam size & $0.1 \mathrm{~mm}$ \\
Slice energy spread & $10 \mathrm{keV}$ \\
Laser wavelength in M1 & $800 \mathrm{~nm}$ \\
Laser wavelength in M2 & $1560 \mathrm{~nm}$ \\
Laser power in M1 & $20 \mathrm{MW}$ \\
Laser power in M2 & $14 \mathrm{MW}$ \\
Laser waist size & $0.4 \mathrm{~mm}$ \\
Undulator period length for M1 & $1.8 \mathrm{~cm}$ \\
Undulator period length for M2 & $2.5 \mathrm{~cm}$ \\
Number of periods for M1 and M2 & 5 \\
Energy modulation in M1 & $30 \mathrm{keV}$ \\
Energy modulation in M2 & $57.4 \mathrm{keV}$ \\
Dispersion strength $R_{56}$ & $14.67 \mathrm{~mm}$ \\
Peak radiation power at $10 \mathrm{THz}$ & $0.33 \mathrm{MW}$ \\
\hline \hline
\end{tabular}

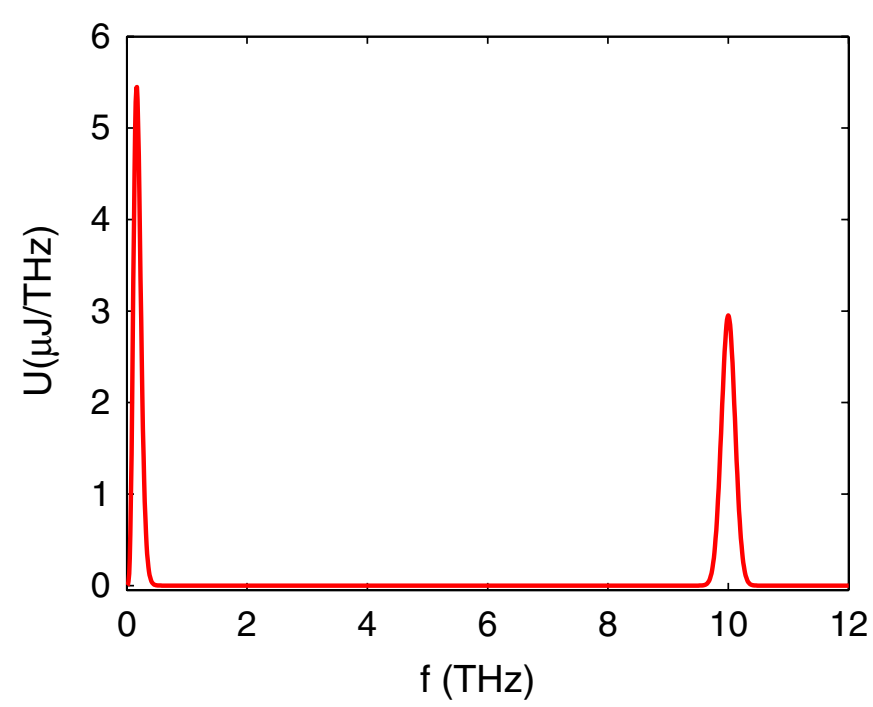

FIG. 7. (Color) Radiation energy density for a laser-modulated electron beam.

tight synchronization with the lasers, it should provide a high temporal resolution for the optical-pump THz-probe experiments. Furthermore, the proposed scheme can be used in energy recovery linac (ERL [6]) to provide high average power $(\sim \mathrm{kW})$ narrow-band $\mathrm{THz}$ radiation which may open up a new regime of $\mathrm{THz}$ sciences not accessible before.

\section{TUNABILITY}

Similar to the DFG scheme, the simplest way to tune the frequency of the $\mathrm{THz}$ radiation in our proposed scheme is to change the laser wavelength. The combination of a Ti:sapphire laser with OPA should allow one to generate $\mathrm{THz}$ radiation covering a wide frequency range using the laser-modulated electron beams. In principle, using a laser 

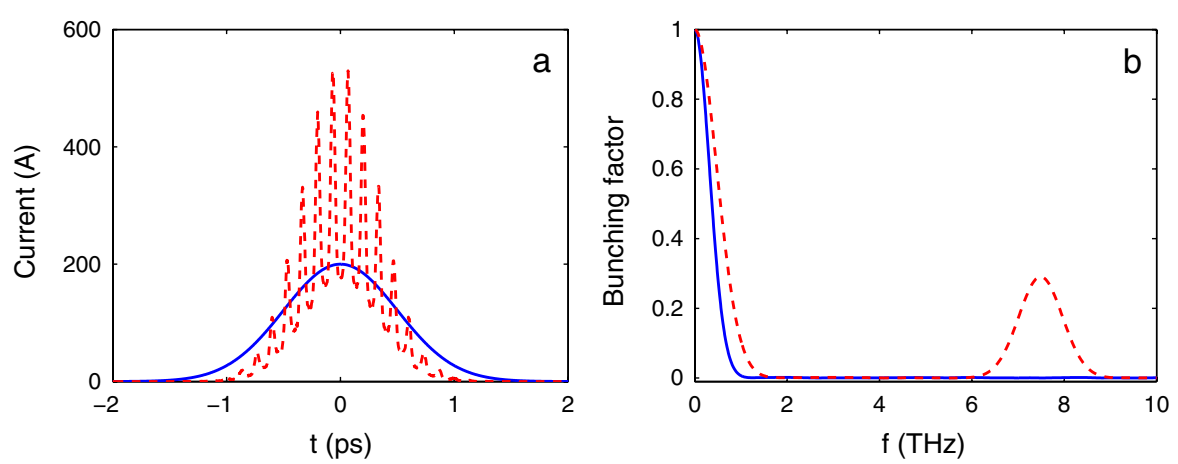

FIG. 8. (Color) (a) Initial current distribution (solid blue line) and that after the dispersion section (dashed red line). (b) Corresponding bunching factor for the initial current distribution (solid blue line) and that after the dispersion section (dashed red line).

with wavelength at $800 \mathrm{~nm}$ and the other laser with wavelength from 1480 to $1600 \mathrm{~nm}$ should allow one to generate $\mathrm{THz}$ radiation covering the whole $\mathrm{THz}$ range.

Another method to tune the $\mathrm{THz}$ radiation frequency in our scheme is through bunch compression or bunch decompression, depending on the sign of the energy chirp factor $h=d p / d \zeta$. Analysis shows in the presence of energy chirp, the wave number shifts to $C\left(n k_{1}+m k_{2}\right)$ (see, for example, [21,22]), where $C=1 /(1+h B)$ is the compression factor. The corresponding optimized bunching factor is found to be

$$
\begin{aligned}
b_{n, m}= & \mid J_{m}\left[C(n+K m) A_{2} B\right] \\
& \times J_{n}\left[C(n+K m) A_{1} B\right] e^{-(1 / 2)[C(n+K m) B]^{2}} \mid .
\end{aligned}
$$

This method allows one to tune the THz frequency even when the laser wavelengths are fixed. Let us still consider the example when the laser wavelengths are 770 and $780 \mathrm{~nm}$ and $C=1.5$ which means the beam is compressed by a factor of 1.5 after passing through the dispersion section. A comparison between Eq. (3) and Eq. (6) implies that one can simply keep the values of $A_{1}$ and $A_{2}$ the same while decreasing $B$ by a factor of 1.5 , then the density modulation is optimized at $7.5 \mathrm{THz}$. In this case, $h \approx$ 0.0184 which corresponds to about $0.45 \mathrm{MeV} / \mathrm{ps}$. The beam current distributions and bunching factors for the energy-chirped beam before and after the dispersion section are shown in Fig. 8.

From Fig. 8, one can see that the macroscopic envelope of the beam is compressed by a factor of 1.5 and the central frequency of the bunching is shifted from 5 to $7.5 \mathrm{THz}$. Using a beam with opposite energy chirp to down-shift the central frequency to lower frequency can be similarly studied.

\section{CONCLUSIONS}

We proposed and analyzed a scheme to generate enhanced narrow-band $\mathrm{THz}$ radiation through downconversion of the frequency of optical lasers using laser- modulated electron beams. The scheme can be understood as DFG of two lasers, with the relativistic electron beam being the nonlinear medium. As compared to the schemes of using ultrashort electron beam to generate $\mathrm{THz}$ radiation, our scheme has the advantage that the $\mathrm{THz}$ radiation is synchronized to the laser and thus allows a higher temporal resolution in optical-pump THz-probe experiments. It can be used in ERL to provide high average power narrow-band $\mathrm{THz}$ radiation which may open up a new regime of $\mathrm{THz}$ sciences not accessible before. The proposed scheme is in principle able to cover the whole $\mathrm{THz}$ range and offers a promising way towards the tunable intense narrow-band $\mathrm{THz}$ sources.

\section{ACKNOWLEDGMENTS}

One of the authors (G. S.) would like to thank S. Milton who attracted his attention to feasibility of using the echo induced beam modulation for generation of $\mathrm{THz}$ radiation. This work was supported by U.S. DOE Contract No. DEAC02-76SF00515.

[1] G. P. Williams, Rep. Prog. Phys. 69, 301 (2006).

[2] K. Reimann, Rep. Prog. Phys. 70, 1597 (2007).

[3] M. Tonouchi, Nat. Photon. 1, 97 (2007).

[4] R. Köhler et al., Nature (London) 417, 156 (2002).

[5] I. Breunig et al., New J. Phys. 10, 073003 (2008).

[6] G. Carr et al., Nature (London) 420, 153 (2002).

[7] G. A. Krafft, Phys. Rev. ST Accel. Beams 7, 060704 (2004).

[8] Y. Shen et al., Phys. Rev. Lett. 99, 043901 (2007).

[9] S. Bielawski et al., Nature Phys. 4, 390 (2008).

[10] J. Neumann et al., J. Appl. Phys. 105, 053304 (2009).

[11] J. Byrd et al., Phys. Rev. Lett. 96, 164801 (2006).

[12] G. Stupakov, Phys. Rev. Lett. 102, 074801 (2009).

[13] D. Xiang and G. Stupakov, Phys. Rev. ST Accel. Beams 12, 030702 (2009).

[14] H. Freund, P. O'Shea, and J. Neumann, Nucl. Instrum. Methods Phys. Res., Sect. A 507, 400 (2003).

[15] I. Blumenfeld et al., Nature (London) 445, 741 (2007). 
[16] P. Muggli et al., Phys. Rev. Lett. 101, 054801 (2008).

[17] L. H. Yu, Phys. Rev. A 44, 5178 (1991).

[18] G. Cerullo and S. De Silverstri, Rev. Sci. Instrum. 74, 1 (2003).

[19] R. Boyd, Nonlinear Optics (Academic Press, San Diego, 2003), 2nd ed.
[20] D. Xiang and W. H Huang, Nucl. Instrum. Methods Phys. Res., Sect. A 248, 163 (2006).

[21] Z. Huang, D. Ratner, G. Stupakov, and D. Xiang, SLACPUB-13547, 2009.

[22] D. Xiang, Z. Huang, and G. Stupakov, Phys. Rev. ST Accel. Beams 12, 060701 (2009). 